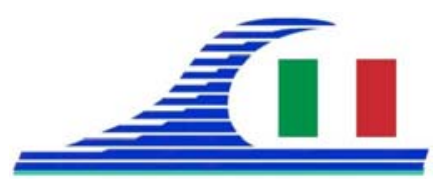

\title{
Le réseau MARocain des Sciences et Ingénierie de la MER (MARSIMER)
}

\author{
Naima HAMOUMI ${ }^{1}$
}

1. Université Mohammed V, Faculté des Sciences, Département de Géologie, Structure de recherche ODYSSEE, Av. Ibn Batouta, BP 1014, Rabat, Maroc. naimahamoumi@yahoo.fr

\section{Résumé :}

Le réseau MARocain des Sciences et Ingénierie de la MER est un réseau thématique multidisciplinaire qui a été mis en place dans le cadre du projet Tempus/Osmose (www.osmose.ma). Il regroupe selon une formule souple les universitaires et les acteurs des secteurs : public, semi public et privé, intervenant dans les espaces littoraux et marins du Maroc. Par ailleurs, il couvre toutes les disciplines de l'océanologie ainsi que le droit et l'histoire de la mer. Ce réseau s'impose en tant que priorité face aux défis et enjeux qui se posent. Il a pour objectifs de cibler les stratégies et les procédures pour:

- développer la coopération à l’échelle locale, nationale, régionale et internationale,

- répondre aux besoins nationaux concernant la recherche, l'innovation et la formation aux métiers de la mer,

- œuvrer pour la promotion des sciences de la mer et le processus de développement durable entrepris par le Maroc,

- contribuer aux efforts internationaux pour la réflexion sur l'avenir de la planète bleue, la préservation des espaces littoraux et marins et la lutte contre le réchauffement climatique,

- donner de la visibilité aux compétences et aux savoir-faire nationaux.

Mots-clés : Réseau, MARSIMER, Sciences et ingénierie de la mer, Recherche, Formation, Médiation scientifique, Maroc.

\section{Présentation et objectifs}

Le réseau MARocain des Sciences et Ingénierie de la MER (MARSIMER) est un réseau thématique multidisciplinaire qui a été mis en place dans le cadre du projet Tempus/Osmose (www.osmose.ma). Il constitue un mécanisme institutionnel pour le développement des partenariats en vue de la mutualisation des moyens et des efforts, ainsi qu'une force d'animation et d'expertise scientifique. En effet, il permet de regrouper selon une formule souple les universitaires et les acteurs des secteurs : public, semi public et privé, intervenant dans les espaces littoraux et marins du Maroc. Par ailleurs, il couvre toutes les disciplines liées à la mer : l’océanologie (géosciences, biologie, sciences halieutiques et aquacoles, physique, chimie, mathématique et 
Côtes méditerranéennes menacées :

Risques et défis dans le contexte du changement climatique

modélisation, météorologie, géodésie, géomatique, ingénierie, etc.), les sciences économiques et juridiques, l'archéologie et l'histoire maritime.

Sa mise en place s'impose en tant que priorité pour le Maroc, pays maritime par excellence, face aux engagements internationaux, aux fondements de la nouvelle constitution et de la loi 01-00 (BO, 2000) et aux défis et enjeux d'ordre scientifique, socio-économique et environnemental, qui se posent. Il a pour objectifs de cibler les stratégies et les procédures pour:

- développer la coopération à l'échelle locale, nationale, régionale et internationale,

- répondre aux besoins nationaux concernant la recherche, l'innovation et la formation,

- développer des formations aux métiers de la mer pertinentes et contribuer ainsi à l'insertion des jeunes dans le tissu socio-économique,

- œuvrer pour la promotion des sciences de la mer et le processus de développement durable entrepris par le Maroc,

- contribuer aux efforts internationaux pour la réflexion sur l'avenir de la planète bleue, la préservation des espaces littoraux et marins et la lutte contre le réchauffement climatique,

- donner de la visibilité aux compétences et aux savoir-faire nationaux.

\section{Mission}

- Contribuer aux politiques, scientifique, environnementale et économique, nationales.

- Euvrer pour la promotion de la gestion intégrée et durable des espaces littoraux et marins, la préservation des ressources vivantes et la sécurité alimentaire.

- Identifier et mener des programmes de recherche et développement et des travaux d'expertises d'intérêt national et régional.

- Mettre en place des formations aux métiers de la mer pertinentes à travers la formation initiale, la formation à la recherche et la formation continue, afin de contribuer à l'insertion des jeunes dans le tissu socio-économique.

- Renforcer les liens entre l’Université et son environnement socio- économique.

- Favoriser l'innovation et la culture de l'entreprenariat.

- Alimenter la base de données LinKup (LINKUP MAROC-COMPETENCES, site web).

\section{Structures}

\subsection{Comité de pilotage}

Le comité de pilotage est la structure décisionnelle chargée de la contribution aux politiques scientifique et environnementale nationales, de la gestion administrative et financière du réseau, de l'élaboration des politiques et des stratégies du réseau concernant les activités de recherche et de formation, la communication, la coopération et les partenariats, ainsi que la valorisation des résultats. 


\subsection{Comité scientifique}

Le comité scientifique assure une mission permanente de suivi de l'exécution des programmes et des actions du réseau. Il est chargé notamment de:

- suivre l'exécution des programmes de recherche et de formation,

- veiller à la valorisation des résultats et à la communication scientifique à travers les publications (articles scientifiques, proceedings, monographies, rapports scientifiques, etc...), à l'alimentation du site web du réseau, à la médiation scientifique, ainsi que l'information et la sensibilisation,

- veiller et alerter sur les enjeux et les risques environnementaux économiques et sociaux,

- proposer et soutenir l'organisation de manifestations scientifiques.

\subsection{Equipes et groupes d'experts}

Les équipes et groupes d'experts réalisent les travaux de recherche, la formation à la recherche et la formation continue, ainsi que les travaux d'expertise scientifique et de consulting. Ils sont également chargés de :

- l'élaboration des programmes de recherche et de formation et des projets de coopération à l'échelle nationale, régionale et internationale,

- la proposition et l'organisation de manifestations scientifiques,

- la valorisation et la publication des résultats,

- la réalisation de bases de données,

- la veille et l'alerte sur les enjeux environnementaux économiques et sociaux,

- la médiation scientifique, ainsi que l’information et la sensibilisation.

\section{Processus de mise en place}

Il s’est échelonné de 2013 à 2014, en plusieurs étapes :

i) Phase préparatoire (8 juillet2013 - 8 septembre 2013) :

- Définition des objectifs et établissement d'un plan d'action pour le réseautage.

- Enquête sur l'état des lieux des sciences de la mer au Maroc.

- Identification des acteurs intervenant dans les espaces littoraux et marins et établissement d'un annuaire pour tous les secteurs : public, semi public, privé.

- Elaboration des outils de travail.

- Campagne d'information et de sensibilisation.

ii) Réseautage des structures de l'université avec leurs partenaires du secteur public et privé (9 septembre 2013- 27 novembre 2013).

iii) Organisation d'une rencontre nationale pour le lancement du réseau le 23 janvier 2013 par la Faculté des Sciences de Rabat, Université Mohammed V-Agdal, le Ministère de l'Equipement et du Transport, l'Institut National de Recherche Halieutique (INRH) et l'Association marocaine pour la Recherche et Développement (R\&D Maroc). 
Côtes méditerranéennes menacées :

Risques et défis dans le contexte du changement climatique

iv) Constitution des organes du réseau (1 mars2014- 9 avril 2014).

v) Elaboration des cadres stratégique, structurel et institutionnel du réseau et de la feuille de route (10 avril 2014- décembre 2014).

\section{Actions et réalisations}

- Participation au nettoyage de la Plage des Oudayas, à Rabat dans le cadre de la 9 ème opération Bahri Dima Clean le 25 mai 2014.

- Organisation du Workshop, "Ensemble pour une gestion intégrée des espaces littoraux et marins marocains", le 6 juin 2015 pour célébrer les événements internationaux dédiés à la mer :

- la Journée mondiale de l’Océan 2015 avec les thèmes "Océan sain, planète saine" et "Un océan en bonne santé, c'est un climat protégé",

- la Journée mondiale de l'Environnement 2015 sur le thème: "Sept milliards de rêves. Une seule planète. Consommons avec modération",

- le vingtième anniversaire de l'entrée en vigueur de la Convention Internationale sur le Droit de la Mer,

- l'année internationale de l'évaluation pour un développement humain équitable et durable.

\section{Logo et contact du réseau}

Pour tout contact, voici le logo et les contacts du réseau MARSIMER :

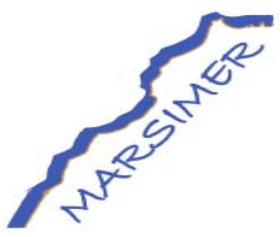

Secrétariat national :

Faculté des Sciences, Université Mohammed V, Av. Ibn Batouta BP 1014 Rabat, Maroc.

Contact : Pr. Naima Hamoumi, coordinatrice nationale, naimahamoumi@yahoo.fr

\section{Références}

LINKUP MAROC-COMPETENCES (site web). http:/osmose.ma/linkup/

BO -Bulletin officiel du Royaume du Maroc- (2000). Loi 01-00 portant sur l'organisation de l'enseignement supérieur. Dahir n 1-00-199 du 15 safar 1421 (19 mai 2000). BO N 4800 du $1^{\text {èr }}$ Juin 2000. 